\title{
Insulin-like growth factor 2 is a key mitogen driving liver repopulation in mice
}

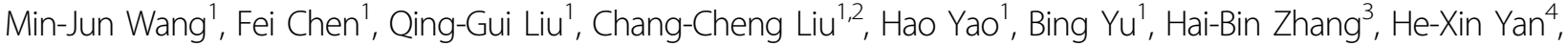 \\ Yibiao Ye ${ }^{5}$, Tao Chen ${ }^{5}$, Kirk J. Wangensteen ${ }^{6}$, Xin Wang ${ }^{7,8,9}$, Yi-Ping Hu ${ }^{1}$ and Zhi-Ying He $\mathbb{C}^{1,2}$
}

\begin{abstract}
Hepatocyte transplantation holds great promise as an alternative to orthotopic organ transplantation in the treatment of liver diseases. However, obtaining clinically meaningful levels of liver repopulation has not been achieved because the mechanisms regulating hepatocyte proliferation in recipient livers have not yet been well characterized. In the mouse model of Hereditary Tyrosinemia Type I, the fumarylacetoacetate hydrolase-deficient $\left(\mathrm{Fah}^{-1-}\right)$ mouse, we found gradually increasing expression level of insulin-like growth factor 2 (IGF2) in the hepatocytes of host livers. Similarly, high levels of IGF2 were found in the livers of patients with deficient FAH activity. Recombinant IGF2 directly promotes proliferation of primary hepatocytes in vitro. Inhibition on IGF2 expression through the interruption of PI3K/Akt and MAPK pathways significantly reduced the level of liver repopulation in $\mathrm{Fah}^{-1-}$ mice. Interestingly, treatment with IGF2 before hepatocyte transplantation generally improved the amount of liver repopulation seen in various mice models of liver injury. Altogether, these findings underscore the underlying mechanisms of therapeutic liver repopulation in $\mathrm{Fah}^{-1-}$ mice, and indicate that IGF2 is a potential hepatocyte mitogen for liver cell transplantation therapies.
\end{abstract}

\section{Introduction}

Cell transplantation therapies have the potential to treat a wide variety of diseases by making up for tissue defects. Several obstacles still hinder the widespread clinical application of cell therapies. Most importantly, the difficulty in achieving sufficient donor cell engraftment into host tissues is one major technical obstacle ${ }^{1}$. Hepatocyte transplantation therapy has been performed in clinical trials as an alternative to orthotopic liver transplantation for some types of genetic diseases of the liver and for acute liver failure ${ }^{2,3}$. However, the extent of liver engraftment and repopulation after hepatocyte

\footnotetext{
Correspondence: Yi-Ping Hu (yphu@smmu.edu.cn) or Zhi-Ying He (zyhe@tongji.edu.cn)

${ }^{1}$ Department of Cell Biology, Center for Stem Cell and Medicine, Second Military Medical University, Shanghai 200433, China

${ }^{2}$ Translational Medical Center for Stem Cell Therapy \& Institute for Regenerative Medicine, Shanghai East Hospital, School of Life Sciences and Technology,

Tongji University, Shanghai 200123, China

Full list of author information is available at the end of the article

Min-Jun Wang, Fei Chen, and Qing-Gui Liu contributed equally to this study.

Edited by $Y$. Wang
}

transplantation was very limited. Therefore, technological improvements to improve therapeutic liver repopulation could lead to successes in cell therapy for liver diseases.

Indeed, therapeutic liver repopulation can be examined under experimental conditions in animal models $^{4-9}$. Two strategies have been successfully applied. The first is to suppress the proliferative capacity of host hepatocytes through inducing cell injuries or damages ${ }^{4-7}$. The second is to supply or regulate hepatic mitogens as well as cell-cycle regulators to drive proliferation of the transplanted hepatocytes in recipient livers ${ }^{8,9}$. Among the rodent models for liver repopulation, the mouse model of Hereditary Tyrosinemia Type I (HT1), the fumarylacetoacetate hydrolasedeficient $\left(\mathrm{Fah}^{-1-}\right)$ mouse, is the best example of repopulation of the liver, reaching $>90 \%$ of total hepatocyte replacement by transplanted wild-type hepatocytes ${ }^{10,11}$.

The liver failure observed in $\mathrm{Fah}^{-1-}$ mouse is similar to what is seen in humans with $\mathrm{HT} 1^{10}$. Loss of FAH results in famarylacetoacetate (FAA) accumulation, a major toxic metabolite, which causes extensive and continuous 
a
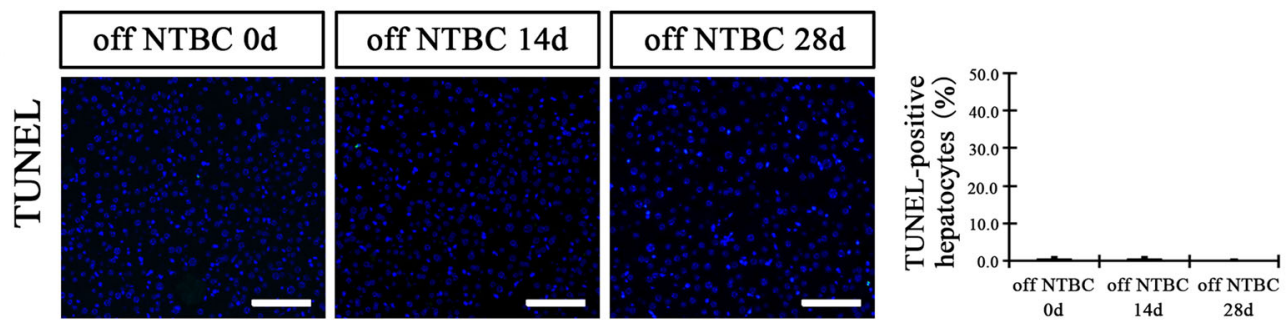

b
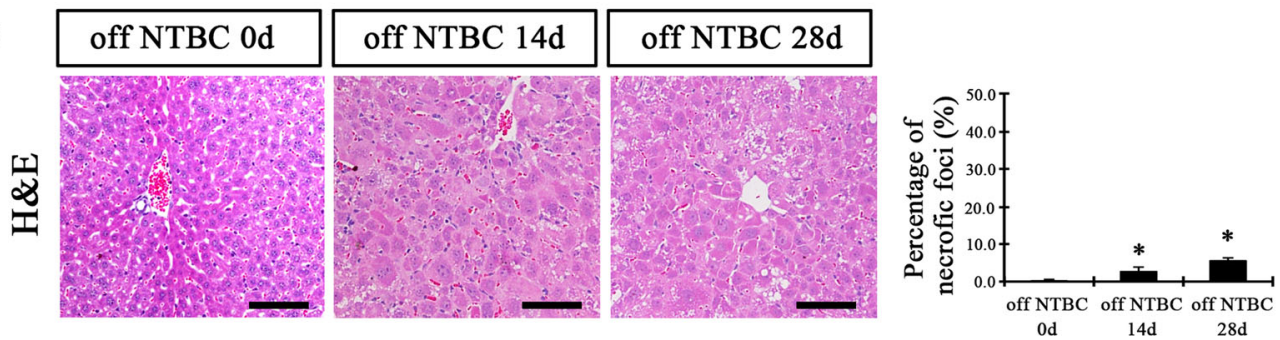

c
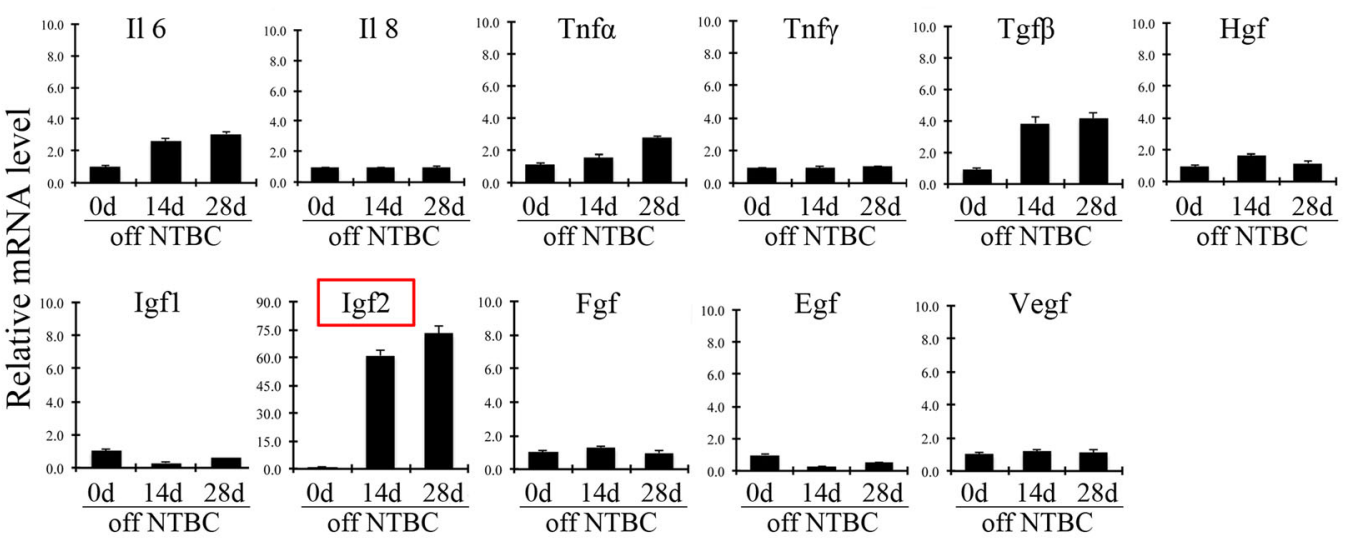

e
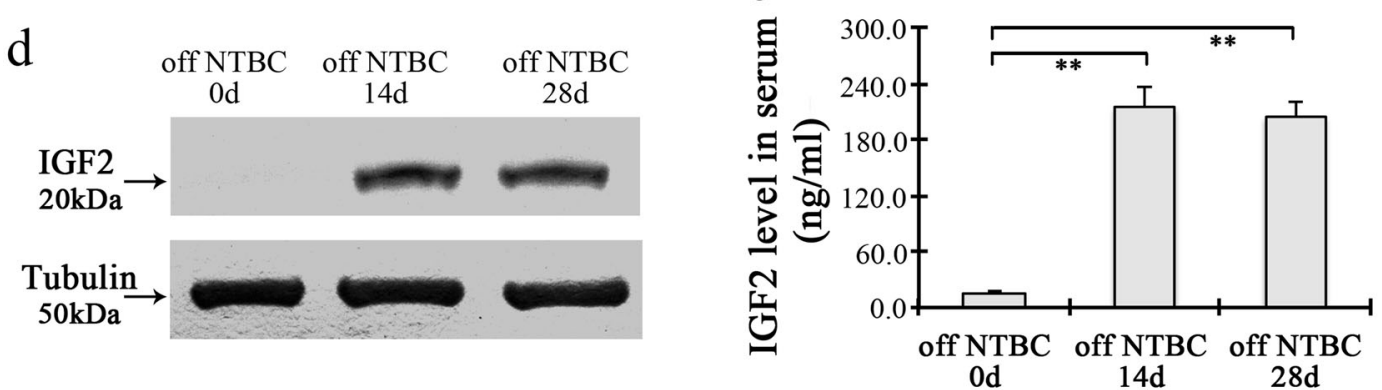

Fig. 1 IGF2 is upregulated during liver injury in Fah $^{-1-}$ mice. Individual panels show the representative photographs for TUNEL staining (a), and hematoxylin-eosin (H\&E) staining (b) in the livers of Fah ${ }^{-1-}$ mice withdrawal of NTBC for 0, 14, and 28 days. The right bar graphs show the quantification of TUNEL-, and necrotic foci-positive cells. c Quantitative RT-PCR of growth factors and cytokines at 0, 14 , and 28 days' livers after NTBC withdrawal. d IGF2 expression levels in liver tissues of Fah ${ }^{-1-}$ mice at 0, 14, and 28 days after NTBC withdrawal. (e) Quantitation of circulating IGF2 levels in $\mathrm{Fah}^{-1-}$ mice at 0,14 , and 28 days after NTBC withdrawal. ${ }^{* *} p<0.01$. Shows are mean \pm S.D. Scale bar, $100 \mu m$

hepatocyte injury. 2-(2-nitro-4-trifluoro-methyl-benzoyl)-1, 3-cyclohexanedione (NTBC) inhibits accumulation of toxic metabolites in hepatocytes to maintain $\mathrm{Fah}^{-1-}$ mice in a healthy state. However, the underlying molecular mechanisms and factors responsible for high repopulation in
$\mathrm{Fah}^{-1-}$ mice still remain elusive and are not well defined. Results from previous studies found that hepatocytes in the livers of $\mathrm{Fah}^{-1-}$ mice undergo DNA damage ${ }^{12}$. Furthermore, a genetic screen has been performed to reveal Foxa3 and TNFR1 as a strong promoter and suppressor of liver 
repopulation in $\mathrm{Fah}^{-1-}$ mice ${ }^{13}$. However, it is not known whether some mitogens are expressed by injured host hepatocytes to enhance the proliferative capacity of transplanted hepatocytes in $\mathrm{Fah}^{-1-}$ mice.

The objective of this study is to carefully elucidate the mechanism of therapeutic liver repopulation in $\mathrm{Fah}^{-1-}$ mouse, which could be used to achieve therapeutic liver repopulation in clinical settings. In the present study, we analyzed the pathological changes in the liver tissues of $\mathrm{Fah}^{-1-}$ mice undergoing injury due to tyrosinemia to discover potential hepatic mitogens which could promote hepatocyte proliferation. We found that the hepatocytes undergoing injury gradually upregulate IGF2 to high levels. Interestingly, IGF2 expression levels return to normal when liver repopulation is completed. Provision of exogenous IGF2 proved it to be an effective mitogen for promotion of proliferation of transplanted hepatocytes. Conversely, inhibition of IGF2 production inhibited repopulation. These findings indicate that IGF2 therapy is a potential strategy promoting liver repopulation in clinical settings.

\section{Results}

IGF2 expression is induced during liver injury in $\mathrm{Fah}^{-/-}$mice

The hepatocytes of $\mathrm{Fah}^{-1-}$ mice undergo injury upon termination of NTBC administration. However, in line with previous reports ${ }^{14}$, we found that only a few scattered hepatocytes become positive for the assay of terminal deoxynucleotidyl transferase-mediated deoxyuridine triphosphate nicked labeling (TUNEL), and only a few small necrotic foci were found in the livers of $\mathrm{Fah}^{-1-}$ mice off NTBC for up to 4 weeks (Fig. 1a, b). These results indicated that there is a lack of cell death of host hepatocytes at the initial stages after hepatocyte transplantation in $\mathrm{Fah}^{-1-}$ mice, implying that hepatic mitogens released by these cells might be responsible for successful liver repopulation in $\mathrm{Fah}^{-1-}$ mice.

Previously, several growth factors and cytokines, such as IL6, TNF $\alpha$, FGF, and HGF, were found to act as mitogens to promote hepatocyte proliferation following partial hepatectomy ${ }^{15-17}$. To test the hypothesis that specific mitogens may promote hepatocyte repopulation of $\mathrm{Fah}^{-1-}$ mice, the candidate factors were investigated in liver tissue by performing RT-PCR at various time points after removal of NTBC (Fig. 1C). Interestingly, Igf2 expression was found to increase 60 -fold at 14 days after withdrawal of NTBC, and increased even more at 28 days. There was a corresponding increase in level of IGF2 protein (Fig. 1d). Remarkably, the level of IGF2 protein in the serum was also found to increase after withdrawal of NTBC (Fig. 1e).

IGF2 is expressed in the hepatocytes of $\mathrm{Fah}^{-/-}$mice and HT1 patients

We examined which cell type in the liver is the major source of IGF2 expression after liver injury. The results indicated that IGF2 was localized to the cytoplasm hepatocytes, and was not detected in other cell types. Furthermore, IGF2 protein was present in the hepatocytes of $\mathrm{Fah}^{-1-}$ mice only after withdrawal of NTBC (Fig. 2a). Moreover, immunohistochemistry assays of repopulated livers demonstrated that IGF2 expression surrounded repopulation nodules in the host liver cells, whereas no expression was detected in FAH enzyme-expressing repopulation nodules (Fig. 2b). Next, DNA synthesis in these $\mathrm{FAH}^{+}$hepatocytes was analyzed through the detection of Ki67 protein on the serially sectioned liver samples. Results revealed that numerous hepatocytes were positive for both Ki67 and FAH inside repopulated nodules, whereas very few of the surrounding host $\mathrm{FAH}^{-}$hepatocytes were positive for Ki67 (Fig. 2c). These data confirm that IGF2 is induced in the injured $\mathrm{FAH}^{-}$deficient host hepatocytes and suggesting that it may act in paracrine fashion on the repopulating, wild-type donor hepatocytes.

Next, we investigated whether IGF2 expression was induced in the humans with liver injury. Whereas few hepatocytes were positive for IGF2 expression in a healthy control, the expression of IGF2 was significantly induced in the hepatocytes of an 8-year-old patient with HT1 (Fig. 2d). These data suggest that increased expression of IGF2 in liver injury may promote liver repopulation.

\section{IGF2 promoted the proliferation of both primary} hepatocytes in vitro and transplanted hepatocytes in vivo

IGF2 was previously reported to act as a hepatocyte mitogen by promoting DNA synthesis ${ }^{18}$. To examine whether IGF2 could affect hepatocyte proliferation directly, we performed a growth assay using wild-type primary hepatocytes cultured with increasing amounts of IGF2 added to the media. The results indicated that 100 and $200 \mathrm{ng} / \mathrm{ml}$ of IGF2 stimulated hepatocyte proliferation significantly (Fig. 3a). Interestingly, this concentration matches the level of IGF2 found in the serum of $\mathrm{Fah}^{-1-}$ mice after NTBC withdrawal. We confirmed stimulated proliferation by performing a BrdU assay, which confirmed that BrdU-positive hepatocytes were significantly increased with IGF2 treatment for $48 \mathrm{~h}$ (Fig. 3b, c). A similar result was found using Ki67 immuno-staining (Fig. 3d, e).

IGF2 is known to bind to insulin-like growth factor receptor 1 (IGF1R), which activates downstream members of PI3K/AKT and mitogen-activated protein kinase (MAPK) pathways ${ }^{19-21}$. To investigate the potential role of IGF2 on liver repopulation, the IGF2 level was analyzed in the repopulated liver of $\mathrm{Fah}^{-1-}$ mice after hepatocyte transplantation. Both mRNA and protein levels of IGF2 increased significantly at the initial stage of liver repopulation within 3 weeks after hepatocyte transplantation (Fig. 4a, b). The expression level then gradually decreased after 3 weeks, and returned to the basal level upon 


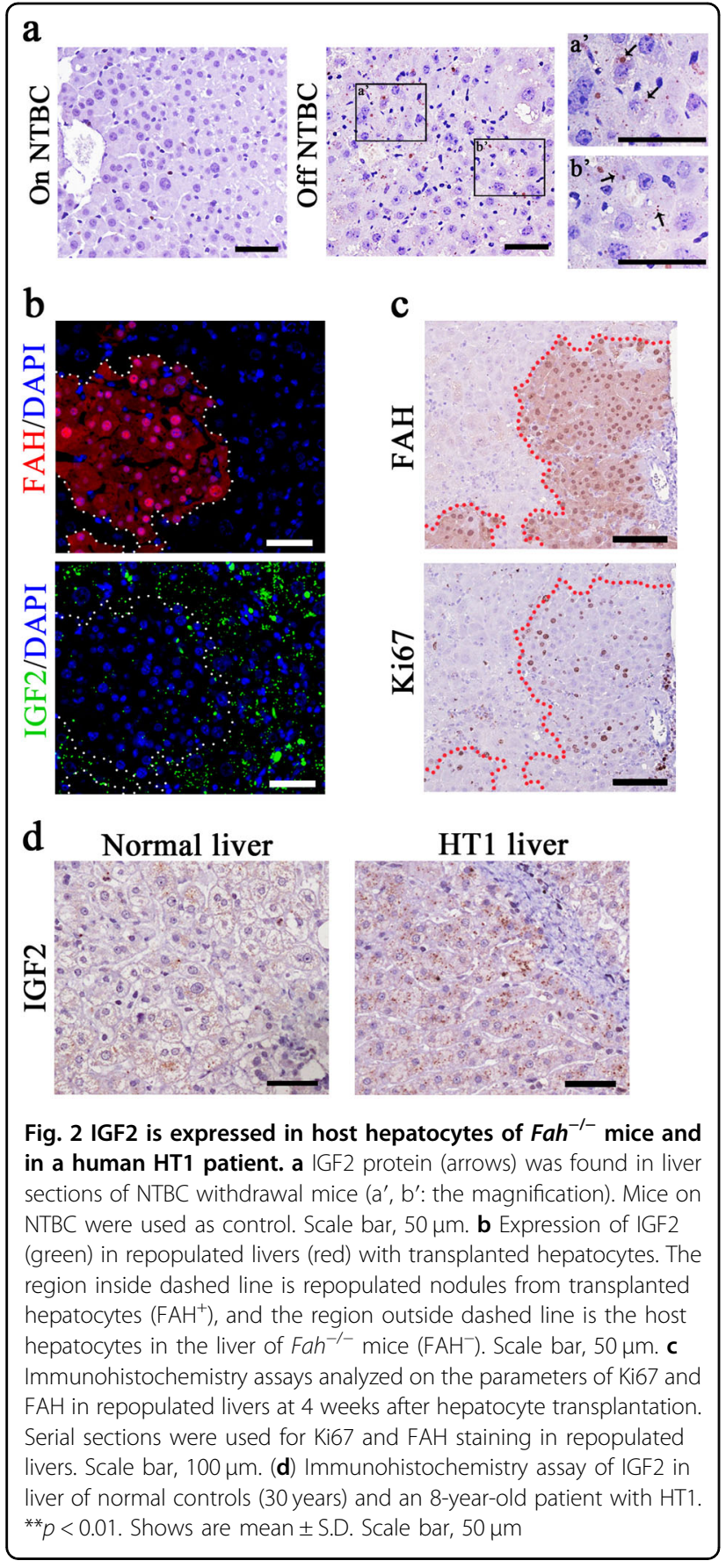

completion of liver repopulation (Fig. 4a, b). Remarkably, there was increased of the phosphorylation of IGF1R and its effectors such as ERK1/2 and AKT in the proliferating hepatocytes, and the levels gradually decreased as liver repopulation went to completion (Fig. 4c-f).

These data indicate that IGF2 is a paracrine signal from the injured hepatocytes that enhances the proliferative capacity of repopulating donor hepatocytes through the activation of AKT and ERK1/2 signaling pathways.
Inhibition of IGF2 expression during hepatocyte transplantation decreased liver repopulation

To examine whether IGF2 expression is required to promote liver repopulation in livers of $\mathrm{Fah}^{-1-}$ mice, we targeted the Igf2 gene using adenovirus vectors expressing shRNA targeting Igf2 (Ad-shIGF2). We used an adenovirus vector expressing GFP gene as a control (Ad-shRNA control). We designed four Ad-shIGF2 vectors, and first tested them in vitro. The Ad-shIGF2 vector with the highest knockdown efficiency was selected for in vivo studies (Supplementary Figure 1).

We injected $\mathrm{Fah}^{-1-}$ mice with Ad-shIGF2 or control after transplantation of donor hepatocytes. Liver samples were harvested at 4,6 , and 8 weeks of repopulation. The levels of both intracellular IGF2 (mRNA and protein) and circulating IGF2 (protein) were significantly decreased in the Ad-shIGF2-treated mice (Fig. 5a-c). Interestingly, the level of liver repopulation observed in the Ad-shIGF2treated mice was substantially reduced at each time point when compared to controls $(7.23 \pm 1.44,19.8 \pm 3.32$, and $44.2 \pm 4.29$ vs. $18.57 \pm 2.81 \%, 64.23 \pm 3.72 \%$, and $85.03 \pm$ $3.29 \%$, respectively, Fig. 5d). We next examined proliferation in the liver tissue and found that the number of Ki67-positive hepatocytes was significantly decreased in the repopulation nodules when IGF2 was knocked down (Fig. 5e). In addition, we investigated the activation level of AKT and ERK1/2 in the two treatment groups. The results indicated that there was a decrease in phosphorylation levels for both AKT and ERK1/2 with IGF2 knockdown, which suggested IGF2 signaling is required for activations of these pathways in repopulating hepatocytes (Fig. 5f).

Collectively, above results indicate that IGF2 enhances liver repopulation with transplanted hepatocytes in $\mathrm{Fah}^{-1-}$ mice.

\section{Promotion of liver repopulation with IGF2 in various animal models}

To study whether supplementation of IGF2 could enhance the proliferative capacity of transplanted hepatocytes and improve liver repopulation, we determined the effect of IGF2 treatment on other models of liver injury, including retrorsine (RS) plus $2 / 3$ partial hepatectomy $(\mathrm{PH})$ or $\mathrm{CCl}_{4}$ treatment. First, the induced mRNA expression for Igf2 along with some other growth factors were analyzed in the livers treated with RS plus $2 / 3$ $\mathrm{PH}$ or $\mathrm{CCl}_{4}$. We found no significantly increased levels of these candidate growth factors including IGF2, indicating that high IGF2 expression may be unique to certain situations such as in HT1 (Supplementary Figure 2).

Next, IGF2 was overexpressed in the hepatocytes of RStreated mice using an adeno-associated virus (AAV)mediated gene delivery system. ROSA ${ }^{\mathrm{mT} / \mathrm{mG}}$ donor 

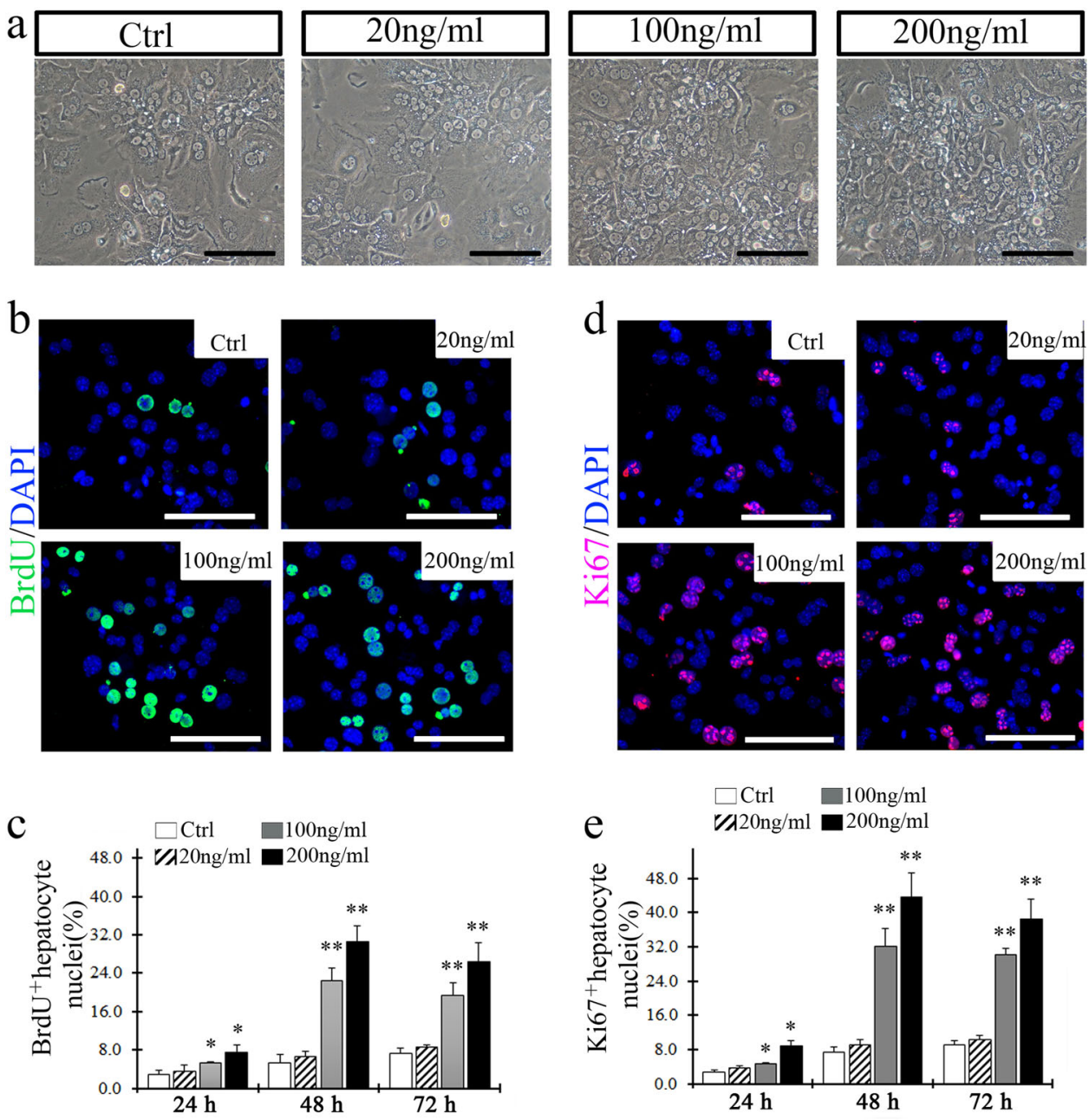

Fig. 3 IGF2 promotes the proliferation of wild-type hepatocytes in culture. a Microscopic images showed the density and morphology for the cultured hepatocytes with or without IGF2 stimuli at $48 \mathrm{~h}$. b Primary hepatocytes were BrdU-positive after treatment with IGF2 for $48 \mathrm{~h}$. c Quantification of BrdU-positive hepatocytes cultured by 24, 48, and $72 \mathrm{~h}$ after stimulation with IGF2 (20, 100, $200 \mathrm{ng} / \mathrm{ml})$. d Ki67-positive primary hepatocytes were found at $48 \mathrm{~h}$ after IGF2 treatment. e Quantification of Ki67-positive hepatocytes. ${ }^{*} p<0.05,{ }^{* *} p<0.01$ vs. cultured hepatocytes without IGF2 stimuli. Shows are mean \pm S.D. Scale bar, $100 \mu \mathrm{m}$

hepatocytes, which were labeled with membrane-localized tdTomato $(\mathrm{mT})$ fluorescence, were transplanted into two groups of mice recipients: (1) mice treated with RS followed by $2 / 3 \mathrm{PH}$; (2) the mice treated with RS followed by $\mathrm{CCl}_{4}$ injection. Four weeks after transplantation, liver samples were harvested and examined for liver repopulation. The results indicated that AAV-mediated IGF2 gene delivery increased the level of liver repopulation by $21.75 \pm 5.91 \%$ in mice treated with RS followed by $2 / 3 \mathrm{PH}$ (Fig. 6a). This occurred via an increase in size of repopulation nodules (Fig. 6a). Similarly, ectopic IGF2 expression increased the level of liver repopulation and the size of repopulated nodules in mice treated with RS followed by $\mathrm{CCl}_{4}$ injection (Fig. 6b). The mTomatopositive donor hepatocytes expressed HNF4 $\alpha$, a hepatocyte marker, during liver repopulation, indicating they are bona fide hepatocytes (Fig. 6c). In addition, numerous of the mTomato-positive hepatocytes were positive for cyclin D1, whereas fewer surrounding host hepatocytes were positive for cyclin D1 (Fig. 6d). Similar results were seen with Ki67 (Fig. 6e).

In summary, overexpression of IGF2 promoted liver repopulation in liver injury models other than $\mathrm{Fah}^{-1-}$ mice. The results also imply that IGF2 could be used to improve liver repopulation in clinical application of hepatocyte transplantation.

\section{Discussion}

Although hepatocyte transplantation is currently considered an experimental alternative to orthotopic liver 

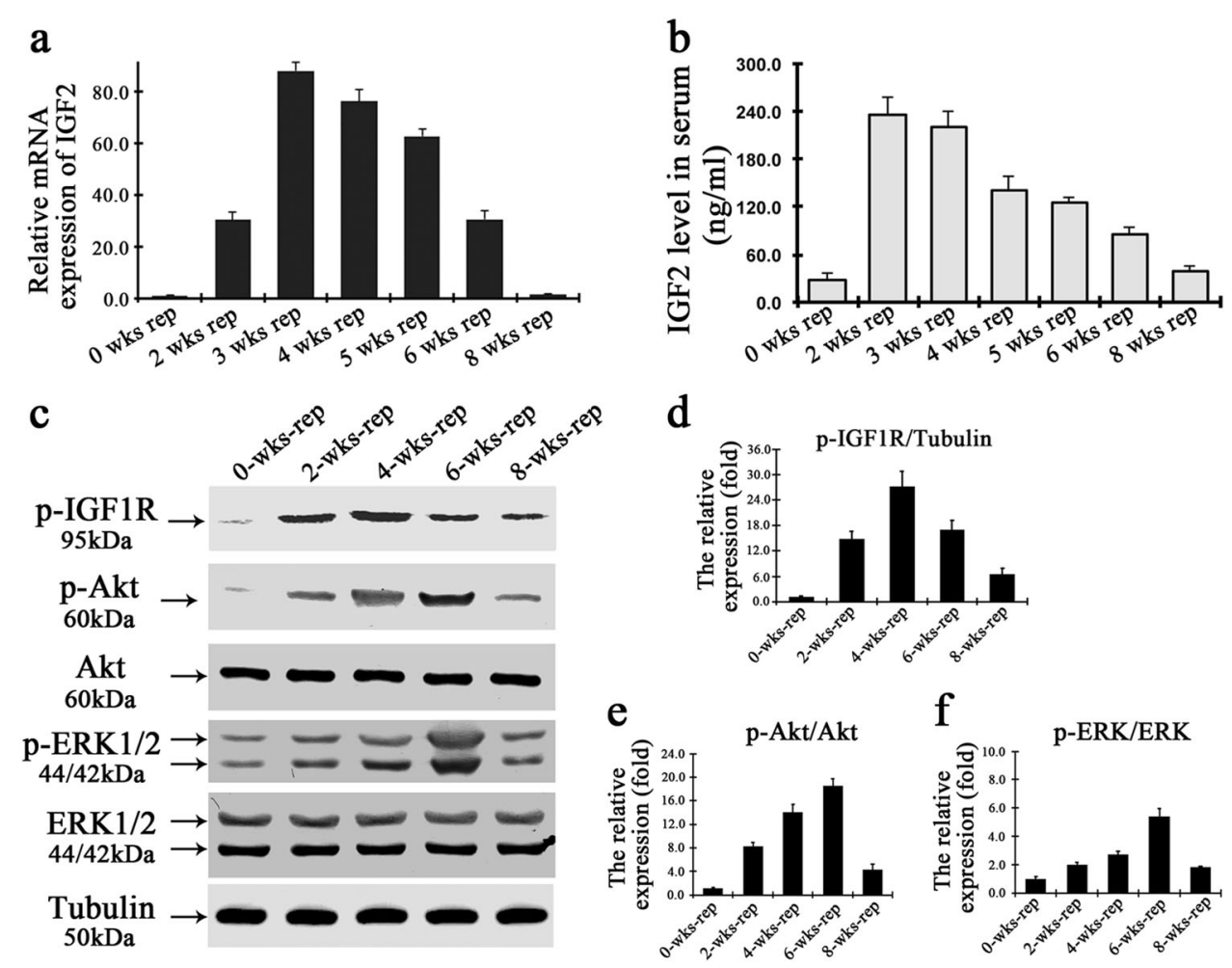

Fig. 4 IGF2 promotes the proliferation of transplanted hepatocytes through activation of PI3K and MAPK pathways. a, b Quantitative assays on the Igf2 mRNA (a) and circulating IGF2 protein (b) in Fah ${ }^{-/-}$mice after hepatocyte transplantation. $\mathbf{c}$ Western blot analysis of phosphorylated IGF1R, extracellular signal-regulated kinase (ERK), and AKT in repopulated livers after hepatocytes transplantation. $\mathbf{d}$ The graph data is the relative level of phosphorylated IGF1R normalized to the inner reference tubulin. e, $\mathbf{f}$ Graphs summarizing relative protein expression levels of phosphorylated AKT (e) or ERK (f) normalized to AKT or ERK, respectively, presented in relative ratios and mean \pm S.D., ** $p<0.01$

transplantation in a few special circumstances, therapeutic levels of liver engraftment repopulation have been hard to reach in most clinical cases. Theoretically, successful liver repopulation will depend on an optimal combination of events, including sufficient blockage of host hepatocyte proliferation in the recipient liver, combined with stimulated proliferation of the transplanted hepatocytes to enable them to win a competition with host hepatocytes for repopulation of the liver, ${ }^{5,22}$. In some patients with acute liver failure, rapid proliferation of transplanted hepatocytes in a short time is the key step for replenishing the missing metabolic capacity. Although repeated transplantation of hepatocytes in large numbers could increase the engraftment of transplanted cells and enhance the liver repopulation, this approach has a risk of thrombosis. In addition, transplanted human hepatocytes must be isolated from unused or rejected donor organs and either used immediately or cryopreserved, therefore there is a shortage of hepatocytes of good quality and enough quantity for transplantation. If the transplanted hepatocytes could be stimulated by mitogenic factors to reach massive levels of repopulation within a short time, the utility in acute liver failure would be tremendous.
Multiple signaling pathways are known to become activated after $\mathrm{PH}$, when including several mitogenic growth factors ${ }^{23}$. Among these, HGF is regarded as a "complete mitogen", as it can induce DNA synthesis during liver regeneration. VEGF promotes hepatocyte proliferation through stimulating HGF production. Some circulating growth factors including EGF and TGF $\alpha$, produced by hepatocytes, stimulate liver regeneration in autocrine or paracrine fashion. TNF as a proinflammatory cytokine mediates hepatocyte apoptosis during $\mathrm{PH}$ induced liver regeneration. However, the mitogenic growth factors identified in PH may not apply to other types of liver "regeneration", such as the liver repopulation that occurs in $\mathrm{Fah}^{-1-}$ mice. In the present study, we examine mitogens in $\mathrm{Fah}^{-1-}$ undergoing liver injury and repopulation, by examining changes in mRNA levels in $\mathrm{Fah}^{-/-}$mouse livers. Among these, IGF2 was to have high levels of induction during liver injury. Interestingly, the expression level of IGF2 increases just slightly during liver regeneration after $\mathrm{PH}^{18,19}$, reflecting differences between $\mathrm{PH}$ and liver injury in $\mathrm{Fah}^{-1-}$ mice. Our study systematically analyzes IGF2 activation during liver injury in $\mathrm{Fah}^{-1-}$ mice, and indicated that IGF2 is a 
a

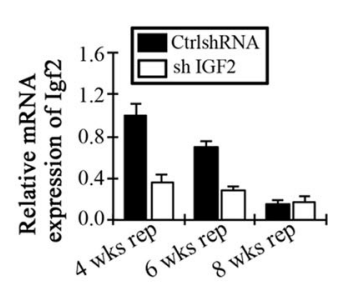

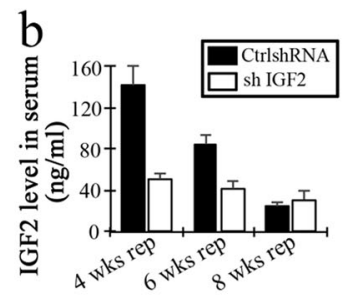

$\mathrm{C}$

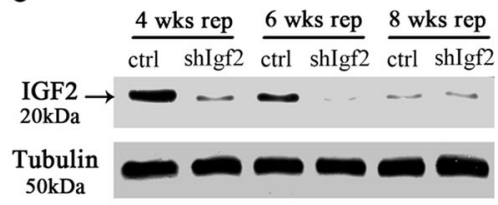

d
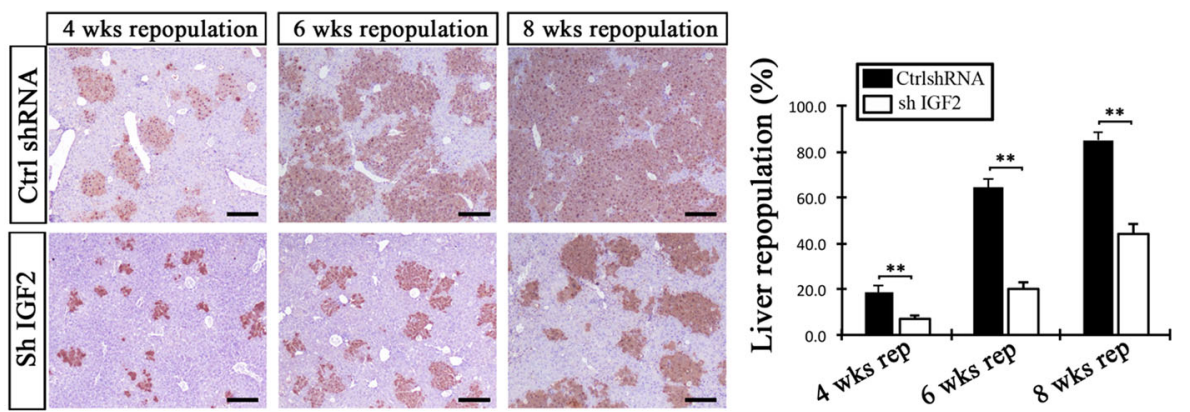

$\mathrm{e}$
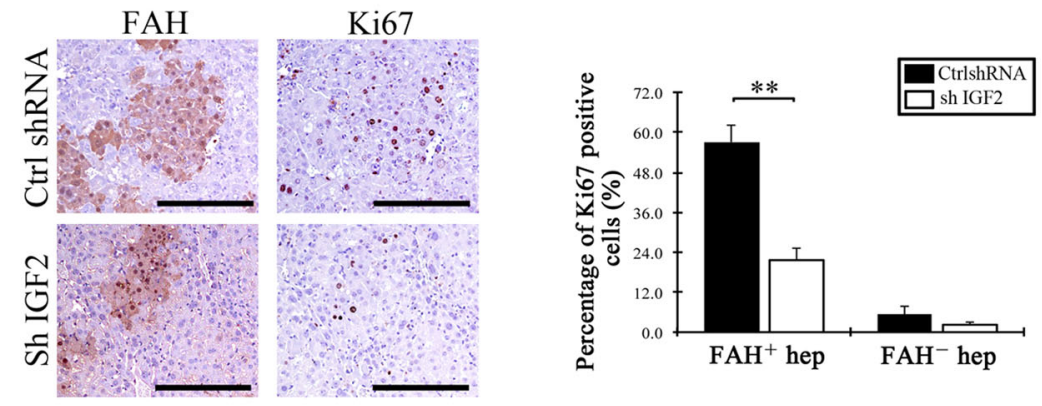

f
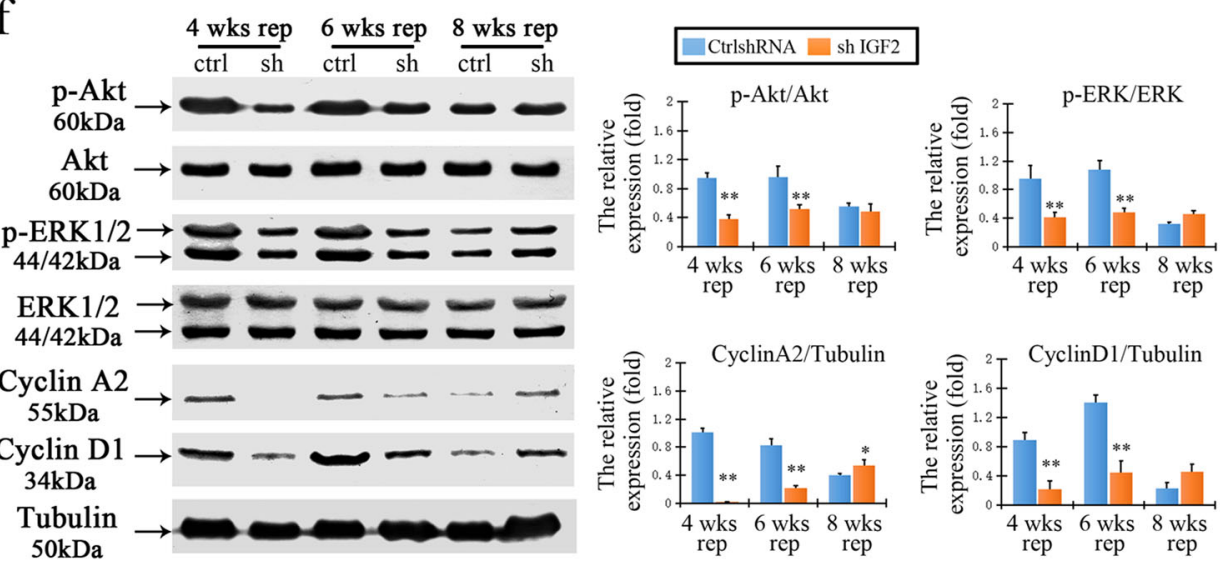

Fig. $\mathbf{5}$ Inhibition of IGF2 expression decreased the levels of liver repopulation. $\mathbf{a}$, $\mathbf{b}$ Levels of IGF2 for both mRNA expression (a) and circulating concentration (b) in the livers of Ad-shlGF2-treated mice decreased after hepatocytes transplantation. c Levels of IGF2 protein expression decreased in the repopulating livers of Ad-shIGF2-treated mice at 2 at 4, 6, and 8 weeks after hepatocyte transplantation. $\mathbf{d}$ Representative sections showed FAH-positive hepatocytes in the repopulated livers at 4, 6, and 8 weeks after hepatocyte transplantation. The right panel showes the proportion of liver repopulation in the Ad-control-shRNA or Ad-shlGF2-treated mice. e The numbers of FAH-positive and Ki67-positive hepatocytes decreased in the livers of mice with 4 weeks of Ad-shIGF2 treatment. The right graph showed the percentage of Ki67-positive cells in both FAH-positive repopulated hepatocytes and FAH negative host hepatocytes after Ad-shlGF2 treatment. $\mathbf{f}$ Levels of p-AKT, AKT, p-ERK1/2, ERK1/2, cyclin A2, and cyclin D1, respectively, in the livers of mice with Ad-shIGF2 treatment at 4,6 , and 8 weeks. The right graphs showed the relative levels of protein expression. Phosphorylated AKT and ERK1/2 were normalized to AKT and ERK1/2 respectively. Cyclin A2 and cyclin D1 normalized to tubulin. Values of Ad-control-shRNA treatment was set as the baseline and considered equal to 1. Data are presented as relative ratios and mean \pm S.D. ${ }^{*} p<0.05$, ${ }^{* *} p<0.01$ vs. Ad-control-shRNA-treated livers. Scale bar, $200 \mu \mathrm{m}$ 

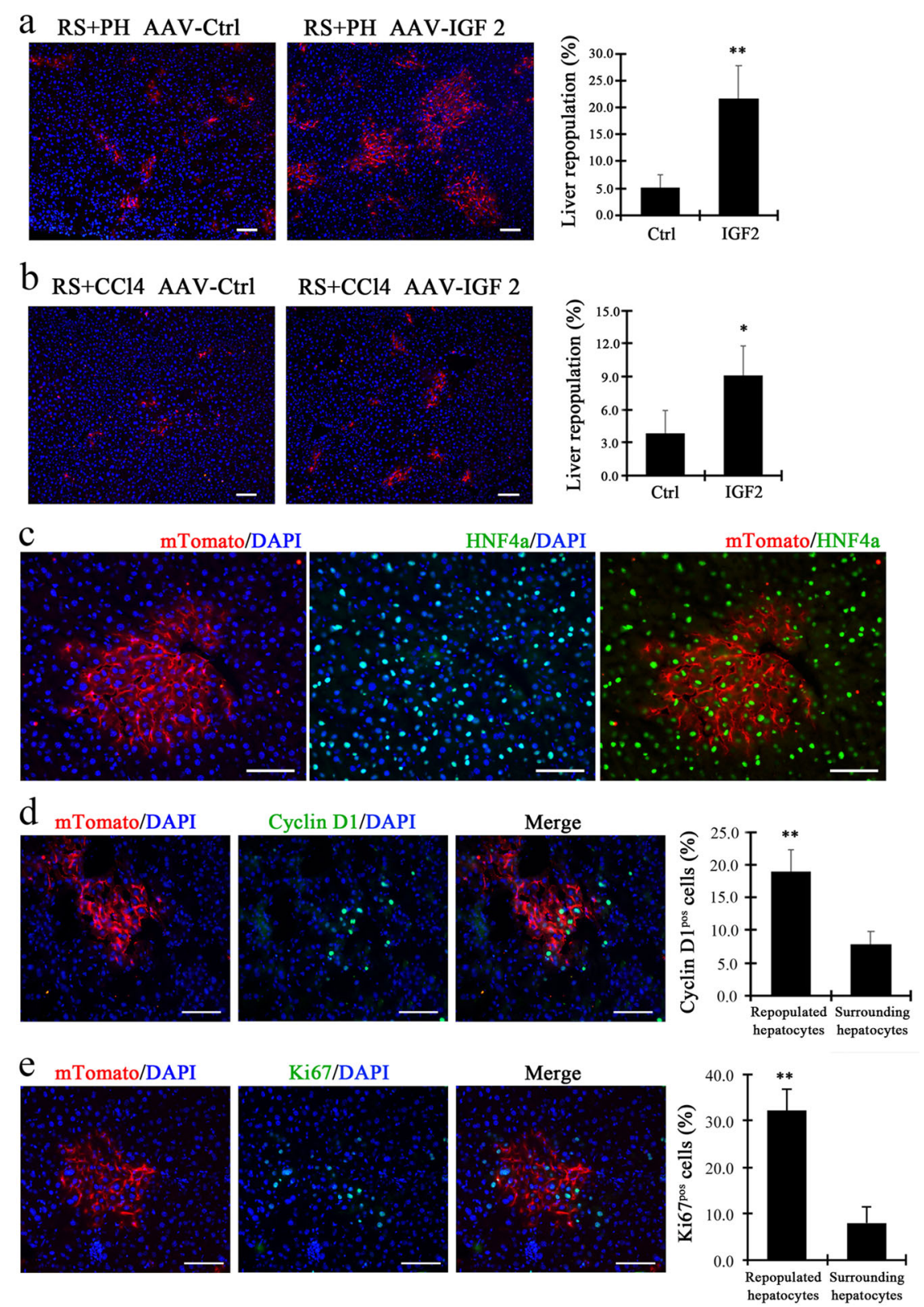

Fig. 6 IGF2 promotes liver repopulation in other mouse models of liver injury. $\mathbf{a}$, b The amounts of ROSA ${ }^{\mathrm{mT} / \mathrm{mG}}$ donor hepatocytes (mTomatopositive) increased in the livers of recipient mice treated with RS followed by $2 / 3 \mathrm{PH}$ (a) or those treated with $\mathrm{RS}$ followed by $\mathrm{CCl}_{4}$ treatment (b). The right panel showed the percentages of mTomato $^{+}$hepatocytes in the livers of mice with or without AAV IGF2 gene delivery. c Co-localization through co-staining with mTomato and HNF4a antibodies indicated the repopulated nodules. $\mathbf{d}$, e Co-localization through co-staining with cyclin D1 (d) or Ki67 (e) and mTomato in repopulated livers after hepatocyte transplantation. ${ }^{*} p<0.05,{ }^{* *} p<0.01$. Shows are mean \pm S.D. Scale bar, $100 \mu \mathrm{m}$

hepatocyte mitogen which promotes liver repopulation. However, other as yet uncharacterized mitogens could also contribute to the promotion on liver repopulation, as shRNA blockade of IGF2 did not fully block the hepatocyte repopulation in $\mathrm{Fah}^{-1-}$ mice. It will be necessary to characterize these as yet unknown hepatocyte mitogens in future studies.

The results from a recent report indicated that short-term treatment with growth hormone improves liver repopulation, which augmented the therapeutic benefit of clinical hepatocyte transplantation ${ }^{24}$. The findings also suggested the possible clinical application of IGF2 during hepatocyte transplantation, which was supported by our findings that overexpression of IGF2 improved liver repopulation in several models of liver injury. In both mouse and human, IGF2 is highly expressed in fetal liver, but the expression level is markedly downregulated in the quiescent adult liver $^{25,26}$ Interesting, it is reported that supplementation of IGF2 into 
the hippocampus of old rats or mice enhances memory and rescues behavioral deficits ${ }^{27,28}$. These data suggest that supplementation of IGF2 could be safe and practical in clinical hepatocyte transplantation.

In summary, host hepatocytes of $\mathrm{Fah}^{-1-}$ mice undergoing injury upregulate IGF2, which stimulates repopulation of the liver by donor hepatocytes. Importantly, IGF2 activates PI3K/AKT and MAPK pathways in transplanted hepatocytes. Our study identifies a key mechanism driving therapeutic liver repopulation in $\mathrm{Fah}^{-1-}$ mice, and suggests a practical strategy to efficiently expand hepatocytes transplanted into receipients in clinical settings.

\section{Materials and methods}

\section{Animals}

$\mathrm{Fah}^{-1-}$ mice (6-8-weeks old) and wild-type donor mice were on a 129S4 background. ROSA-mTomato/mGFP $\left(\mathrm{ROSA}^{\mathrm{mT} / \mathrm{mG}}\right)$ reporter mice and wild-type recipients were maintained on a C57BL/ 6 background. $\mathrm{Fah}^{-1-}$ mice were maintained with or without $7.5 \mathrm{mg} / \mathrm{l}$ 2-(2-nitro-4trifluoromethylbenzoyl)-1, 3-cyclohexanedione (NTBC) in the drinking water ${ }^{10,11}$. Human liver tissue was obtained from a surgical resection specimen of a patient with a hepatic hemangioma and a patient with HT1, and consent from the patients were obtained. All procedures and protocols were according to institutional guidelines.

\section{Hepatocyte isolation, cell transplantation, and repopulation assay}

Mouse livers were perfused with collagenase D (Roche, Indianapolis, IN) solution as previously described ${ }^{29,30}$. Isolated hepatocytes were injected intrasplenically into recipient mice as described previously ${ }^{29}$. After hepatocytes transplantation, livers were harvested and immunohistochemistry with FAH antibody or mTomato was used to examine the percentage of liver repopulation as described previously ${ }^{29}$.

\section{Experimental liver treatments}

For retrosine-based 2/3 PH injury model ${ }^{31}$, C57BL/6 wild-type mice were given three injections of retrorsine (Sigma-Aldrich, St. Louis, MO), $30 \mathrm{mg} / \mathrm{kg}$ each, intraperitoneally, 3, 2, and 1 weeks before the transplantation. 1 day before the transplantation, recipient mice were subjected to a two-thirds partially hepatectomy $(\mathrm{PH})$. For retrosine-based $\mathrm{CCl}_{4}$ injury ${ }^{32}$, $\mathrm{C} 57 \mathrm{BL} / 6$ recipient mice were intraperitoneally infused with $0.6 \mathrm{ul} / \mathrm{g}$ carbon tetrachloride $\left(\mathrm{CCl}_{4}\right.$, Sigma-Aldrich $) 1$ day before the transplantation.

\section{Primary hepatocyte culture and BrdU incorporation}

Isolated primary hepatocytes were plated on collagencoated 6-well plates in Hepatic Cell Culture Medium
(Invitrogen, Carlsbad, CA). Six hours after seeding, nonadherent cells were removed by washing the plated with phosphate buffered saline and the attached cells were then cultured in conditional hepatic cell culture medium supplemented with $0,20,100$, and $200 \mathrm{ng} / \mathrm{ml}$ recombinant IGF2 (R\&D Systems, Minneapolis, MN). For BrdU incorporation assay, cells were incubated for $24 \mathrm{~h}$ with BrdU regent (final concentration $100 \mu \mathrm{M}$ ) (SigmaAldrich). Then, cells were fixed with a 1:1 mixture of icecold methanol:glacial acetic acid after washing with PBS, and then incubated with $2 \mathrm{~N} \mathrm{HCl}$ for $30 \mathrm{~min}$ at room temperature. Then cells were incubated with primary and second antibody as with the IHC protocols.

\section{Construction adenoviruses expressing lgf2 shRNA}

Small hairpin RNA (shRNA) sequences for the Igf2 gene were chosen from Sigma-Aldrich and listed as follows: Igf2 shRNA-1:5'-CCGGGCTTGTTGACACGCTTCAGT TCTCGAGAACTGAAGC

GTGTCAACAAGCTTTTTG-3', 5'-AATTCAAAAA GCTTGTTGACACGCTTCAGTTCT

CGAGAACTGAAGCGTGTCAACAAGC-3'; Igf2 sh RNA-2: 5'-CCGGGTGGGCAAGT

TCTCCAATATCTCGAGATATTGGAAGAACTTGC CCACTTTTTG-3', 5'-AATTCAA

AAAGTGGGCAAGTTCTTCCAATATCTCGAGATA TTGGAAGAACTTGCCCAC-3';

Igf2 shRNA-3: 5'-CCGGCTGATCGTGTTACCACCCAAACTCGAGTTTGGGTGGT

AACACGATCAGTTTTTG-3', 5'-AATTCAAAAACT GATCGTGTTACCACCCAAACT

CGAGTTTGGGTGGTAACACGATCAG-3'; Igf2 sh RNA-4: 5'-CCGGCAAAGA

\section{GTTCAGAGAGGCCAACTCGAGTT}

GGCCTCTCTGAACTCTTTGTTTTTG-3', 5' -AATTCAAAAACAAAGAGTTCAGAGAGGCCAACTCGAGT TGGCCTCTCTG

AACTCTTTG3'. The annealed oligonucleotides were ligated into the pLKO.1 vector and their sequences were verified. Then the four different Igf2 shRNAs were recombined into an adenoviral vector (AdEasy system) to generate recombinant adenovirus vector that expresses shRNA for Igf2, as previously described. Adenoviruses were generated by transfecting HEK 293A cells with vectors digested with PacI according to the manufactuer's instructions. Viruses were purified on a discontinuous $\mathrm{CsCl}$ gradient. Ad-shIGF2 expression plasmids were transfected into the hepa1- 6 cell line to test the knockdown efficiency of these IGF2 shRNA constructs. The AdshIGF2 with the highest knockdown efficiency was selected for in vivo experiments. Mice received adenovirus via tail-vein injection at a dose of $1 \times 10^{9}$ viral particles per gram body weight. A recombinant adenovirus vector 
that expresses a shRNA against GFP (Ad-shGFP) was used as a negative control.

\section{AAV8-IGF2 design and in vivo delivery}

The IGF2 overexpression and control adeno-associated virus were generated by Obio Technology (Shanghai, China). The purified virus was diluted in sterile PBS. Viruses $\left(5 \times 10^{11}\right)$ were injected through tail-vein at the volume of $100 \mu \mathrm{l} 3$ weeks before cell transplantation.

\section{Statistical analysis}

All experiments were performed at least three times. Data were expressed as the mean \pm standard deviation (s. d.). Statistical analyses were carried out using Graph Pad Prism 5.0c for Mac (Graph Pad Software). For parametric data, data significance was analyzed using a two-tailed unpaired Student's $t$-test. In cases where more than two groups were being compared, then a one-way ANOVA was used. $F$-tests were used to compare variances between groups. $p<0.05$ was considered statistically significant.

\section{Acknowledgements}

This work was funded by National Natural Science Foundation of China (81471948, 81772954, 31271474, 31601101, and 31271469), Shanghai Committee of Science and Technology of China (15JC1403900), Science and Research Fund of Shanghai Municipal Commission of Health and Family Planning (20164Y0013), Shanghai Sailing Program (17YF1424400), National Key Basic Research and Development Program of China (2011CB966200, 2010CB945600), Shanghai Pujiang Program(17PJD045), and Science and Technology Development Fund Of Shanghai Pudong New Area (PKJ2017-Y16).

\section{Author details}

${ }^{1}$ Department of Cell Biology, Center for Stem Cell and Medicine, Second Military Medical University, Shanghai 200433, China. ${ }^{2}$ Translational Medical Center for Stem Cell Therapy \& Institute for Regenerative Medicine, Shanghai East Hospital, School of Life Sciences and Technology, Tongji University, Shanghai 200123, China. ${ }^{3}$ Eastern Hepatobiliary Surgery Hospital, Second Military Medical University, Shanghai 200433, China. ${ }^{4}$ Department of Anesthesia and Intensive Medicine, Renji Hospital Affiliated to Shanghai Jiaotong University School of Medicine, Shanghai 200127, China. ${ }^{5}$ Department of Hepatobiliary Surgery, Sun Yat-sen Memorial Hospital, Sun Yat-sen University, Guangzhou, China. ${ }^{6}$ Department of Medicine, Division of Gastroenterology, University of Pennsylvania, Philadelphia, PA 19104, USA. ${ }^{7}$ The Key Laboratory of National Education Ministry for Mammalian Reproductive Biology and Biotechnology, Inner Mongolia University, Huhhot 010070, China. ${ }^{8}$ Department of Laboratory Medicine and Pathology, University of Minnesota, Minneapolis, MN 55455, USA. ${ }^{9}$ Hepatoscience Incorporation, 725 San Aleso Avenue, Sunnyvale, CA 94085, USA

\section{Authors contributions}

Z.Y.H. and Y.-P.H. conceived and supervised the project. M.-J.W., F.C., and Q.-G.L. performed the experiments and interpreted data. C.-C.L., and H.Y. performed animal experiments. H.-B.Z. and H.-X.Y. provided human samples. B.Y., Y.Y., and T.C. assisted in the experiments. X.W. provided constructive comments on the project. M.-J.W., K.J.W., X.W., and Z.-Y.H. wrote the manuscript. All authors discussed the results and commented on the manuscript

\section{Conflict of interest}

The authors declare that they have no conflict of interest.

\section{Publisher's note}

Springer Nature remains neutral with regard to jurisdictional claims in published maps and institutional affiliations.

Supplementary Information accompanies this paper at https://doi.org/ 10.1038/s41419-017-0186-1.

Received: 16 August 2017 Revised: 2 November 2017 Accepted: 27 November 2017

Published online: 18 January 2018

\section{References}

1. Hill, E., Boontheekul, T. \& Mooney, D. J. Regulating activation of transplanted cells controls tissue regeneration. Proc. Natl Acad. Sci. USA 103, 2494-2499 (2006).

2. Soltys, K. A. et al. Barriers to the successful treatment of liver disease by hepatocyte transplantation. J. Hepatol. 53, 769-774 (2010).

3. Huebert, R. C. \& Rakela, J. Cellular therapy for liver disease. Mayo Clin. Proc. 89, 414-424 (2014).

4. Shafritz, D. A. \& Oertel, M. Model systems and experimental conditions that lead to effective repopulation of the liver by transplanted cells. Int. J. Biochem. Cell Biol. 43, 198-213 (2011).

5. Oertel, M., Menthena, A., Dabeva, M. D. \& Shafritz, D. A. Cell competition leads to a high level of normal liver reconstitution by transplanted fetal liver stem/ progenitor cells. Gastroenterology 130, 507-520 (2006).

6. Menthena, A. et al. p15INK4b signaling, and cell competition promotestem/ progenitor cell repopulation of livers in aging rats. Gastroenterology 140, 1009-1020 (2011).

7. Serra, M. P. et al. Hepatocyte senescence in vivo following preconditioning for liver repopulation. Hepatology 56, 760-768 (2012).

8. Karnezis, A. N., Dorokhov, M., Grompe, M. \& Zhu, L. Loss ofp27 (Kip1) enhances the transplantation efficiency of hepatocytes transferred into diseased livers. J. Clin. Invest. 108, 383-390 (2001).

9. Xiang, D. et al. Non-viral FoxM1 gene delivery to hepatocytes enhances liver repopulation. Cell Death Dis. 5, e1252 (2014).

10. Overturf, K. et al. Hepatocytes corrected by gene therapy are selected in vivo in a murine model of hereditary tyrosinaemia type I. Nat. Genet. 12, 266-273 (1996).

11. Overturf, K., al-Dhalimy, M., Ou, C. N., Finegold, M. \& Grompe, M. Serial transplantation reveals the stem-cell-like regenerative potential of adult mouse hepatocytes. Am. J. Pathol. 151, 1273-1280 (1997).

12. Paulk, N. K. et al. In vivo selection of transplanted hepatocytes by pharmacological inhibition of fumarylacetoacetate hydrolase in wild-type mice. Mol. Ther. 20, 1981-1987 (2012).

13. Wangensteen, K. J., Zhang, S., Greenbaum, L. E. \& Kaestner, K. H. A genetic screen reveals Foxa3 and TNFR1 as key regulators of liver repopulation. Genes Dev. 29, 904-909 (2015).

14. Vogel, A. et al. Chronic liver disease in murine hereditary tyrosinemia type 1 induces resistance to cell death. Hepatology 39, 433-443 (2004).

15. Yoshiya, S. et al. Blockade of the apelin-APJ system promotes mouse liver regeneration by activating Kupffer cells after partial hepatectomy. J. Gastroenterol. 50, 573-582 (2015).

16. Padrissa-Altés, S. et al. Control of hepatocyte proliferation and survival by Fgf receptors is essential for liver regeneration in mice. Gut 64, 1444-1453 (2015).

17. Kaldenbach, M. et al. Hepatocyte growth factor/c-Met signaling is important for the selection of transplanted hepatocytes. Gut 61, 1209-1218 (2012).

18. Kimura, M. \& Ogihara, M. Effects of insulin-like growth factor I and II on DNA synthesis and proliferation in primary cultures of adult rat hepatocytes. Eur. J. Pharmacol. 354, 271-281 (1998).

19. Mogler, C. et al. Hepatic stellate cell-expressed endosialinbalancesfibrogenesis and hepatocyte proliferation during liver damage. EMBO Mol. Med 7, 332-338 (2015).

20. Lamonerie, T., Lavialle, C., Haddada, H. \& Brison, O. IGF-2 autocrine stimulation in tumorigenic clones of a human colon-carcinoma cell line. Int. J. Cancer 61, 587-592 (1995). 
21. Foulstone, E. et al. Insulin-like growth factor ligands, receptors, and binding proteins in cancer. J. Pathol. 205, 145-153 (2005).

22. Krause, P., Rave-Frank, M., Christiansen, H. \& Koenig, S. Preconditioning of the liver for efficient repopulation by primary hepatocyte transplants. Methods Mol. Biol. 1213, 29-39 (2014).

23. Kang, L. I., Mars, W. M. \& Michalopoulos, G. K. Signals and cells involved in regulating liver regeneration. Cells 1, 1261-1292 (2012).

24. Stock, P. et al. Impairment of host liver repopulation by transplanted hepatocytes in aged rats and the release by short-term growth hormone treatment. Am. J. Pathol. 187, 553-569 (2017).

25. Lui, J. C., Finkielstain, G. P., Barnes, K. M. \& Baron, J. An imprinted gene network that controls mammalian somatic growth is down-regulated during postnatal growth deceleration in multiple organs. Am. J. Physiol. Regul. Integr. Comp. Physiol. 295, R189-R196 (2008).

26. Lui, J. C. \& Baron, J. Evidence that Igf2 down-regulation in postnatal tissues and upregulation in malignancies is driven by transcription factor E2f3. Proc. Natl Acad. Sci. USA 110, 6181-6186 (2013).
27. Chen, D. Y. et al. A critical role for IGF-\|l in memory consolidation and enhancement. Nature 469, 491-497 (2011).

28. Pascual-Lucas, M. et al. Insulin-like growth factor 2 reverses memory and synaptic deficits in APP transgenic mice. EMBO Mol. Med 6, 1246-1262 (2014).

29. Wang, M. J. et al. Reversal of hepatocyte senescence after continuous in vivo cell proliferation. Hepatology 60, 349-361 (2014).

30. He, Z. Y. et al. Murine embryonic stem cell-derived hepatocytes correct metabolic liver disease after serial liver repopulation. Int. J. Biochem. Cell Biol. 44, 648-658 (2012).

31. Nagamoto, Y. et al. Transplantation of a human iPSC-derived hepatocyte sheet increases sunvival in mice with acute liver failure. J. Hepatol. 64, 1068-1075 (2016).

32. Turner, R. A. et al. Successful transplantation of human hepatic stem cells with restricted localization to liver using hyaluronan grafts. Hepatology $\mathbf{5 7}, 775-784$ (2013). 